\title{
MOVIMIENTOS SOCIALES Y NUEVAS DEMANDAS POLÍTICAS: EL MOVIMIENTO POR LA PAZ *
}

\author{
JAIME PASTOR \\ Profesor Titular de Ciencia Política \\ UNED
}

* Este artículo es una versión de la comunicación presentada con el mismo título por el propio autor al III Congreso de Sociología, celebrado en septiembreoctubre de 1989. 
Revista de Derecho Político, núm. 34, 1991, pp. 225-235

\title{
MOVIMIENTOS SOCIALES Y NUEVAS DEMANDAS POLÍTICAS: EL MOVIMIENTO POR LA PAZ
}

\author{
POR \\ JAIME PASTOR \\ Profesor Titular de Ciencia Política
}

UNED

La aparición de lo que se ha dado en llamar «nuevos movimientos sociales" constituye desde hace tiempo objeto de estudio dentro de las ciencias sociales. Parece haber un relativo consenso respecto a su significación como expresión de nuevos valores y demandas políticas, relacionadas con la lucha por la extensión de una «tercera generación» de derechos y libertades y con el empleo de formas de participación política no convencional. Partiendo de ese acuerdo inicial se ha podido comprobar que muchos de esos movimientos se han ido configurando como grupos de presión en el marco de los respectivos sistemas políticos, e incluso han llegado a transformarse en algunos casos en nuevos partidos políticos. Todo esto va a ser contrastado con la experiencia del movimiento por la paz en el Estado español, con el fin de valorar las distintas etapas que ha vivido y las particularidades que su propio caso representa.

Conviene, sin embargo, partir antes de una breve caracterización de esos movimientos. Para ello el enfoque empleado por Claus OFFE tiene, en mi opinión, bastante utilidad en muchos aspectos. Ași, si bien existen diferencias entre las razones y objetivos finales específicos de cada movimiento, cabe reconocer que las causas de su emergencia y desarrollo tienen dos raíces comunes, al menos en el área de los paises occidentales. La primera sería la crisis del concepto de seguridad, tal como fue entendido 
desde 1945, y la segunda estaría relacionada con la crisis de los sistemas de partidos o de la política institucional en general.

- Así, desde finales del decenio de los setenta se puede observar una crisis de la idea de seguridad entendida en tres dimensiones: la material, ofrecida por el Estado del bienestar; la militar, proporcionada por la disuasión nuclear, y la de «orden público», basada en la eficacia del control social de lđ población. De manera más o menos aguda, según los casos, se puede decir que los movimientos feminista, pacifista y ecologista se han apoyado en la quiebra de esas tres dimensiones y en el malestar social que ha generado en determinadas capas de la población, especialmente entre trabajadores del sector servicios con un nivel educativo alto, grupos sociales «desmercantilizados» e incluso capas medias rurales. Los límites de la política de bienestar han hecho más visible la crisis de un modelo de crecimiento económico y sus efectos en el ecosistema planetario, así como viejas y nuevas desigualdades de clases, de sexos o de razas; asimismo, la instalación de los euromisiles reveló la dinámica suicida de la disuasión nuclear $y$, por consiguiente, la creación de una mayor inseguridad frente al teórico enemigo ${ }^{\text {. }}$

En cuanto a la crisis del sistema de partidos, se observa también una actitud de distanciamiento respecto al mismo por parte de capas sociales que lo consideran simple expresión de una democracia competitiva elitista e incapaz de recoger su malestar social y las demandas que sugieren los nuevos movimientos sociales, convertidos en verdaderos actores políticos que rebasan los marcos estrechos de cada Estado-nación. Esto explica que, llegados a cierto grado de desarrollo, en núcleos organizados de esos movimientos se haya planteado la necesidad de transformar lo que inicialmente eran grupos de presión en nuevos partidos políticos, después de haber vivido experiencias basadas en alianzas conflictivas con partidos tradicionales.

En cualquier caso, la tendencia a reconocer legalmente los nuevos valores y derechos (igualdad entre sexos y razas, protección de la naturaleza y paz positiva, por ejemplo) no significa que se hayan puesto los medios para su consecución efectiva, ya que ello supondría la búsqueda de nuevos modelos de crecimiento económico y de organización de la sociedad a escala transnacional; tampoco se puede considerar que la presión a favor de una democracia participativa, exigida por esos movimientos, se haya abierto paso en los sistemas políticos democrático-liberales salvo, quizás, mediante un reconocimiento legal más flexible de mecanismos

1 En ese sentido es importante recordar que los acuerdos de desarme parcial han favorecido un aumento de las tendencias contrarias a la OTAN y a la presencia norteamericana en Europa dentro de los principales países de la CEE, como demuestran los eurobarómetros (véase Ronald INGLEHART, Cultural Change in the Advanced Industrial Society, Princeton Univ., 1989, capítulos 11 y 12). 
como la iniciativa legislativa popular, el recurso a los referéndum sobre materias tradicionalmente excluidas de la consulta popular (aunque en esto se haya avanzado poco) o la objeción de conciencia.

Entrando en el caso particular del movimiento por la paz, tiene también interés indicar que si bien el motivo principal de su irrupción a finales de los años setenta fue la denuncia de la amenaza de guerra nuclear, pronto la evolución de los grupos organizados de ese movimiento ha ido más lejos, llegando a enlazar con las más profundas tradiciones del pacifismo moderno, surgido alrededor de la crítica al monopolio estatal del uso de la violencia política, interna y externa y, por lo tanto, de la denuncia del hecho histórico de que la democracia no haya sido capaz de penetrar en esas esferas que, como se sabe, constituyen la última ratio de los Estados. Esto tiene su importancia porque explica, como recuerda GALTUNG ${ }^{2}$, la razón de ser estratégica del movimiento por la paz y, al mismo tiempo, la enorme dificultad que entraña una tarea semejante.

El movimiento por la paz en el Estado español comparte en su corta historia los dos rasgos que antes se han presentado en el área occidental: la critica a la idea de seguridad vinculada a la política de bloques militares y la defensa del derecho a una paz positiva, por un lado; la presión por una democracia participativa, en relación conflictiva con los principales partidos del sistema político, por otro. Pero conviene hacer una breve cronología de este proceso.

Así, en primer lugar, es importante no olvidar que antes del comienzo de la transición política existian ya grupos organizados de pacifistas, basados en la defensa de la objeción de conciencia al servicio militar obligatorio como actividad fundamental. En esta etapa se puede decir que su relación con los partidos era casi nula, si bien no cabe subestimar su impacto entre sectores cristianos ${ }^{3}$. De cualquier manera, ya bajo el fran-

2 Véase, por ejemplo, J. GaLtung, "Los nuevos movimientos sociales y la izquierda actual", en A. GuerRa et alii, El nuevo compromiso europeo, Madrid, Sistema, 1987.

3 Se puede encontrar una breve historia del movimiento hasta 1985 en Carlos IRIART, "Apuntes para una memoria del movimiento pacifista español en 1985", en Anuario sobre Armamentismo en España, 1986, Centro de Investigación para la Paz (CIP), Madrid-Barcelona, Fontamara, 1986. 
quismo se reflejaban algunos rasgos de la opinión pública en los que se va a apoyar el pacifismo español: neutralismo histórico y antiamericanismo.

Pero el punto de partida de la irrupción del movimiento por la paz como tal se halla, sin duda, en las reacciones frente al comienzo de la «segunda guerra fría» y ante la decisión del gobierno Calvo Sotelo de entrar en la OTAN después del 23-F. Se puede sostener, por lo tanto, que en los años 81-82 surge un movimiento débilmente organizado, pero con un amplio consenso entre la población y con el apoyo público de la principal fuerza electoral de izquierdas, el PSOE. En ese bienio se configura una opinión pública en la que, junto al neutralismo histórico y al antiamericanismo, vemos expresarse un rechazo a la política de bloques militares y a las armas nucleares. Las expectativas que abre la victoria electoral socialista de octubre del 82 favorecen, además, las esperanzas en un cambio no sólo del sistema de partidos sino también del rumbo que habia adoptado hasta entonces la Reforma Política, mediante la superación del «síndrome del 23-F” y la ampliación de la democracia.

Entre los años 82 y 85 vemos transcurrir un proceso en el que la inicial alianza entre el grupo de presión (el movimiento por la paz) y el principal partido del sistema (el PSOE) se va transformando én un enfrentamiento creciente en dos aspectos: en la forma de entender el pacifismo y en la vía de resolución de la decisión definitiva sobre la OTAN. En el primer aspecto, es bien sabido que hay un cambio de actitud por parte del PSOE, que considera compatible la defensa del derecho a la paz con la permanencia en el bloque militar occidental; en el segundo, la promesa de referéndum se ve postergada hasta después de la entrada en la CEE y se convoca la "consulta" cuando la presión de la opinión pública a favor de su realización se revela más fuerte que todas las reticencias de las élites políticas y militares.

En el periodo citado, sobre todo en los años 84 y 85 , se produce la consolidación de los núcleos organizados del movimiento por la paz, así como su amplia coordinación territorial, apoyados sin duda en el amplio consenso ganado en la opinión pública ${ }^{4}$. Pero sus soportes dentro del sistema de partidos se ven sensiblemente reducidos, después de la defección

4 Conviene recordar que en 1983 empieza a constituirse la Coordinadora Estatal de Organizaciones Pacifistas (CEOP) y en marzo de 1985 se celebran las Jornadas de debate de Barcelona, a partir de las cuales se intensifica la campaña a favor de un referéndum con una pregunta clara. Sobre el consenso logrado entre la opinión pública, son bastante ilustrativos los estudios de Francisco Alvira («Opinión Pública y Defensa en España", diciembre de 1988) o las sucesivas encuestas del $\mathrm{CIS}$ en ese periodo (véase "Actitudes y opiniones de los españoles ante las relaciones internacionales", CIS, septiembre 1987; también, A. IzQUIERdo Escribano, "La conciencia pacifista española: un aporte estadístico", en Anuario sobre Armamentismo en España, 1987-1988, CIP, Madrid, Debate, 1988). 
del PSOE. No obstante, combinando acciones de calle con iniciativas de recogida de firmas ${ }^{5}$, la conquista de la convocatoria del referéndum aparece, en ese contexto de ascenso, como la victoria de una concepción de la democracia participativa basada en el empleo de formas de actuación política no convencional, claramente defendida por los partidarios del NO a la OTAN y sólo finalmente aceptada por el gobierno.

Sin embargo, el primer trimestre de 1986 marca un cambio de tendencia fundamental: el contenido mismo de la pregunta del referéndum, la utilización extremadamente desigual de los medios de comunicación y los sucesivos argumentos empleados por el líder del partido gobernante para justificar el Sí no son contrarrestados por el limitado tejido social organizado del pacifismo, con lo cual se modifica la opinión de sectores decisivos del electorado, se produce la derrota del movimiento y se legitima la incorporación definitiva a un bloque militar. Así, lo que podía aparecer como un referéndum antihegemónico se transforma finalmente en un referéndum pro-hegemónico, hasta el punto que se llega a concluir, en opinión de muchos, que la democracia elitista se ha visto reforzada por otra de tipo plebiscitario ${ }^{6}$.

El desenlace de la consulta no afecta, por lo tanto, únicamente a la permanencia en un bloque militar. Tiene implicaciones en la democracia misma, ya que no supone la apertura de una nueva fase de mayor parti-

5 Es importante recordar el apoyo social que encuentran las diversas campañas de recogida de firmas a favor del referéndum, a pesar de que no tenían ninguna fuerza legal, debido al carácter restrictivo de la regulación de la iniciativa legislativa popular y a la exclusión de ella de materias de carácter internacional. Se trata, sin duda, de otro ejemplo de participación política no convencional durante este corto período, reflejado a su vez en el carácter masivo de las acciones convocadas por la CEOP y en la disposición a vincularse bajo distintas formas a los grupos pacifistas antiatlantistas, como se demuestra también en sondeos realizados por OTR/IS y CIS durante los años 83-85.

6 Véase, por ejemplo, el estudio del Equipo de Sociología Electoral «EI referéndum del 12 de marzo de 1986 sobre la permanencia de España en la OTAN y sus consecuencias para el sistema político», en Revista de Estudios Políticos, 52, julio-agosto 1986; también, Javier Pérez Royo, «Repercusiones del referéndum en el sistema politico español», en F. G. Gil y J. S. Tulchin (eds.), España y la OTAN. Perspectivas Politicas y Estratégicas, Madrid, ICl, 1987. Sobre la influencia de los medios de comunicäción, principalmente la TV, y su contraste con la de los grupos primarios y los líderes de opinión, me remito al estudio de A. IzQUIERDo, basado en las encuestas del CIS posteriores al referéndum, titulado «La OTAN en casa: familia y televisión en el referéndum» y publicado en En Pie de Paz, 7, septiembre-octubre 1987. Conviene resaltar asimismo el hecho de que la actitud abstencionista de la derecha representada por AP favorece también la campaña del gobierno ante un sector del electorado de izquierdas. Por otra parte, en cuanto a los resultados del referéndum, no cabe olvidar que el NO es mayoritario en Euskadi, Catalunya y Canarias. 
cipación política por parte de los ciudadanos. La causa de ello podría encontrarse en el hecho de que la derrota del NO a la OTAN se convierte en un factor de frustración política para las capas sociales con un nivel de información y de cultura mayor que han constituido, en gran parte, el verdadero soporte del movimiento. El adelantamiento de las elecciones generales y la fragilidad de las fuerzas políticas afines al pacifismo antiatlantista facilitan, además, el distanciamiento respecto al sistema político de unos o el retorno al voto tradicional de otros ${ }^{7}$.

Probablemente sólo en el caso de una victoria del NO en el referéndum se podría haber planteado la hipótesis de creación de un partido de nuevo tipo, basado en un agrupamiento de las principales corrientes presentes en el movimiento coalicionista que se había formado. Sin embargo, incluso en ese caso las dificultades habrían sido importantes, teniendo en cuenta las diferencias políticas entre aquéllas (izquierda comunista tradicional, izquierda radical, izquierda nacionalista y pacifismo no violento) y la todavía débil red de miembros organizados con que contaba el movimiento, por no mencionar la menor extensión de lo que en países como la RFA se llama «sector público voluntario». Pero, de cualquier manera, se trata de una especulación que ya no tiene sentido y que sólo se plantea en aquel momento en sectores muy reducidos.

Asi, tras marzo del 86, el movimiento por la paz se ve obligado a reformular sus objetivos. En su ámbito especifico se producen debates sobre la estrategia a seguir respecto a las promesas encerradas en el preámbulo de la pregunta del referéndum (no estructura militar, reducción presencia norteamericana, no armas nucleares), mientras que en el político general se revelan diferentes respuestas respecto a las vías de expresión electoral del NO a la OTAN, quebrándose asi la relativa unidad del movimiento.

Pero el incumplimiento de las promesas no ha provocado nuevas movilizaciones con fuerza comparable a las vividas en el período 82-86; tampoco los modestos resultados electorales de las pocas fuerzas políticas favorables al movimiento han permitido que éste logre un apoyo sustancial

7 Basta recordar que mientras un 38 por 100 de los antiguos votantes del PSOE desoyeron las recomendaciones de voto de su partido en el referéndum, una vez celebrado éste, sólo el 8 por 100 anunciaba su intención de votar a otra formación política, mientras que un 32 por 100 estaba indeciso (Julián Santamaria y Mercedes ALcover, Actitudes de los españoles ante la OTAN, CIS, septiembre 1987). También habría que estudiar las consecuencias de la frustración política de un sector social significativo en la aparición de un nuevo tipo de abstención, más politizado, en las posteriores convocatorias electorales (este aspecto aparece indirectamente apreciado en varios estudios de actitudes ante los partidos y respecto a la participación electoral; véase, por ej., la comunicación de Joan FONT y Paloma FONTCUBERTA «Participación política versus participación electoral», en el III Congreso de Sociología). 
dentro del sistema de partidos. El movimiento por la paz ha perdido así el protagonismo central que obtuvo en lo que algunos consideran «la última batalla de la transición", entrando en una nueva etapa en la que las labores de contrainformación y fomento de la investigación para la paz pasan a primer plano, no sin abandonar por ello la preparación de marchas y acciones periódicas que tienen ya cierta tradición en su corta historia, o la organización de actividades concretas frente a la presencia de barcos nucleares, el comercio de armas o la instalación de nuevos campos de tiro. En cuanto a su función como grupo político de presión, no cabe duda que ha conocido un debilitamiento sustancial, fomentado también por la beligerancia de los principales medios de comunicación frente a un movimiento al que consideran prácticamente deslegitimado tras el 12 de marzo del 86.

Naturalmente, dentro de las confusas perspectivas a corto plazo no cabe duda que influye el nuevo panorama que ofrece una coyuntura internacional en la que los acuerdos de desarme parcial han devuelto la iniciativa a las grandes potencias y ante las cuales la opinión pública se encuentra a la expectativa de cuál sea la reformulación de la política de disuasión nuclear.

Pero es importante resaltar el hecho de que el reflujo en las acciones callejeras del movimiento no significa que se haya reducido la simpatía de amplios sectores de la opinión pública: los sondeos realizados tras el referéndum muestran un descenso lógico de la sensación de amenaza de guerra nuclear, pero mantienen una desconfianza frente a los bloques militares, así como el rechazo de la presencia militar norteamericana y de las armas nucleares. Cabe añadir que es precisamente el nuevo contexto internacional el que favorece una actitud más reticente frente a los gastos militares y ante la institución militar en general ${ }^{8}$. Por eso sería un error deducir de la menor presencia pública del movimiento pacifista un retroceso en el interés de un notable sector del electorado por las cuestiones de política exterior y defensa, si bien éstas parecen tender a plantearse en el contexto de la CEE y de los debates sobre las características que debería tener la defensa europea ${ }^{\circ}$.

8 Me remito al estudio ya citado de Francisco Alvira, quien recuerda la existencia de una opinión mayoritaria que considera excesivo el gasto en Defensa, que hay otras prioridades y que no hay un enemigo inmediato que justifique esos gastos.

${ }^{\circ}$ Es interesante mencionar, según una encuesta del CIS de julio de 1988 sobre "Construcción de Europa y Defensa», que existe una opinión mayoritaria contraria a las siguientes opciones: enviar fuerzas militares a otros paises europeos, aceptar que tropas de otros paises de la Alianza Atlántica se estacionen en España, permitir que la marina y la aviación de guerra de nuestros aliados utilicen las instalaciones militares españolas, y permitir que algunas unidades militares estén mandadas ocasionalmente por mandos extranjeros. Sólo un $\mathbf{4 0}$ por 100, frente a un 36 por 100 , es partidario de la participación en maniobras militares en colaboración con otros países. 
La tendencia indicada antes respecto a la actitud ante los gastos militares enlaza con una cuestión polémica que hoy está planteando un sector del movimiento por la paz. Me refiero a la objeción de conciencia al servicio militar obligatorio y al rechazo de la prestación social sustitutoria por parte de un número modesto pero significativo de jóvenes. Se trata de un fenómeno de especial interés, ya que supone la impugnación de un deber impuesto históricamente por muchos Estados-naciones y que hoy aparece obsoleto tanto ante las características que adoptan los Ejércitos contemporáneos y sus estrategias como frente a la extensión de una conciencia pacifista que considera rechazable la preparación para la guerra frente a un presunto enemigo.

No faltan ya propuestas procedentes de algunos de los principales partidos en las que se pretende responder a esa actitud con la alternativa del servicio militar voluntario, cuya adopción plantearía nuevos problemas y tampoco daría satisfacción al sector de objetores insumisos. Pero parece difícil la implantación de esta medida a corto plazo, por razones que no corresponde analizar ahora ${ }^{10}$. Mientras esto no sea cambiado, nos encontramos con un problema fundamental para el sistema político español y para el poder judicial en particular: ¿cómo responder a la extensión de la desobediencia civil al servicio militar y a la prestación social sustitutoria por razones políticas e ideológicas y no únicamente religiosas? En la actualidad, la Ley de Objeción de Conciencia ha demostrado ser un marco excesivamente restrictivo para legalizar la situación de miles de jóvenes que se niegan a hacer la «mili» en nombre de valores considerados superiores 0 , simplemente, por su repudio a la imagen tradicional y a la función de control social autoritario del ejército español. Por eso, en esta cuestión aparece un nuevo reto al sistema político, máxime cuando esas acciones no se plantean ya como actitudes puramente individuales sino como declaraciones colectivas que afectan a muchos jóvenes. Un problema semejante podría plantearse en el futuro con la objeción fiscal a los gastos de defensa por parte de un número no despreciable de contribuyentes ${ }^{11}$.

Como conclusión, se puede sostener que los valores pacifistas se encuentran ya arraigados en sectores amplios de la opinión pública, si bien

10 Sobre los argumentos igualitaristas empleados por responsables del Ministerio de Defensa para justificar el mantenimiento del servicio militar obligatorio, véase A. Pérez Henares et alii, Luces y Sombras del Poder Militar en España, Madrid, Ediciones Temas de Hoy, 1989, cap. III.

11 Puede verse un estudio de la campaña de objeción fiscal y una relación de las organizaciones de carácter pacifista o humanitario beneficiadas en En Pie de Paz, 12, enero-marzo 1989. 
existen distintas interpretaciones respecto a la forma de relacionar esos valores con la búsqueda de políticas alternativas frente a la disuasión nuclear, a la permanencia de los bloques militares o a los problemas que va a plantear una política de defensa europea. En la actualidad esa cultura pacifista está conduciendo a una actitud crítica hacia los gastos de Defensa y a una impugnación del servicio militar obligatorio, tanto por tratarse de una institución heredada de un modelo de Estado-nación también en crisis como por la menor verosimilitud de las amenazas exteriores en la coyuntura internacional en que nos encontramos.

Respecto a la relación del movimiento por la paz con el sistema de partidos, hemos visto que ha atravesado distintas etapas: la inicial alianza se ha visto sustituida por una tendencia a la confrontación con los principales partidos, pero sin que el movimiento por la paz haya tenido la suficiente fuerza y unidad para favorecer la aparición de un nuevo tipo de formación capaz de ser su expresión política en el marco de las instituciones. En cuanto al precedente que ha significado el uso de una técnica de democracia semi-directa como el referéndum para zanjar el problema de la presencia en un bloque militar, ya se ha recordado los límites que tuvo esa consulta para estimular una nueva dinámica de participación política tanto por el desarrollo de la campaña como por su resultado. Así pues, en estos momentos el desafio al sistema político se plantea en términos más concretos: se trata de valorar hasta qué punto es compatible el mantenimiento del principio de la legitimidad de las leyes, en función de su aprobación por mayorias parlamentarias, con el reconocimiento de acciones de desobediencia civil frente a determinadas decisiones de política exterior y de defensa consideradas ilegítimas en nombre de la defensa prioritaria de los valores de la paz y la supervivencia. De la eficacia del movimiento por la paz para crear un apoyo social a ese tipo de acciones y de su repercusión en el sistema político depende el futuro de ese movimiento.

Cabria analizar también hasta qué punto el protagonismo alcanzado por el movimiento por la paz puede ser sustituido en el futuro por un movimiento ecologista que, estimulado por su auge en el área europea, llegue incluso a contar con una formación política presente en las instituciones. Pero es aún pronto para hacer hipótesis en este sentido, debido a la juventud y divisiones de ese movimiento en el caso español, si bien las recientes elecciones europeas demuestran que existen expectativas razonables para que ese proceso dé buenos frutos. Probablemente sean las elecciones municipales, debido a sus propias características, las que ofrezcan mayores oportunidades para juzgar las posibilidades que tiene el intento de dotar de un brazo político a ese movimiento. 